\title{
UNA RELACIÓN ENTRE EL TRABAJO INFANTIL Y DESERCIÓN ESCOLAR
}

\author{
Julio César Zavala Umanzor, Universidad Nacional Autónoma de Honduras (UNAH), \\ Facultad de Ciencias Económicas, Administrativas y Contables (FCEAC), \\ Instituto de Investigaciones Económicas y Sociales (IIES), \\ Ciudad universitaria, edificio C2, primer piso, Tel/fax: (504) 2239-1849 \\ E-mail: zavalajulio@iiies-unah.org \\ José Roberto Arrazola Raudales, Universidad Nacional Autónoma de Honduras (UNAH), \\ Facultad de Ciencias Económicas, Administrativas y Contables (FCEAC), \\ Instituto de Investigaciones Económicas y Sociales (IIES), \\ Ciudad universitaria, edificio C2, primer piso, Tel/fax: (504) 2239-1849 \\ E-mail: jarrazola@iies-unah.org
}

\section{RESUMEN}

EL trabajo infantil es una actividad que atenta con el esparcimiento emocional de los niños, sin embargo, es un evento latente en la sociedad hondureña y, si bien es cierto el trabajo infantil no es completamente nocivo per se, pero las casusas que lo motivan lo envuelven en un problema y las condiciones en las que se realiza son las agravantes al mismo. Muchas instituciones a nivel internacional y nacional velan por que los niños gocen de libertades y beneficios, y ante la imperante necesidad de que trabajen, consideran normas para clasificar las labores como permitidas para los infantes. Por otro lado, la educación es un derecho que se ve muchas veces truncado, al igual que el trabajo infantil, por diversos factores, ante ésta situación el estudio merece importancia, pues se pretende explorar una relación entre el trabajo infantil y la deserción escolar, el trabajo es un inicio para desarrollar la temática, pues sugiere un estudio por separado entre el dominio rural y urbano de este tema. Encontrando una relación a priori entre la población infantil que labora y la que desierta de la educación, en el periodo de 2007 a 2012 en Honduras.

Palabras Clave: trabajo infantil, niños, deserción escolar, educación. 
Vol. 4

$\mathrm{N}^{\circ} 2$

2013

E\&A

IIES

162

\title{
A RELATION BETWEEN CHILD LABOR AND SCHOOL DECERSION
}

\author{
Julio César Zavala Umanzor, Universidad Nacional Autónoma de Honduras (UNAH), \\ Facultad de Ciencias Económicas, Administrativas y Contables (FCEAC), \\ Instituto de Investigaciones Económicas y Sociales (IIES), \\ Ciudad universitaria, edificio C2, primer piso, Tel/fax: (504) 2239-1849 \\ E-mail: zavalajulio@iies-unah.org
}

\begin{abstract}
ABSTRAC
Child labor is an activity that attentive with the recreation emotional well-being of children, however, it is a latent event in Honduran society and, if it is true that the child labor is not completely harmful per se, but the sec-ondary causes underlying envelop you in a problem and the conditions under which it performs are the aggravating at the same. Many institutions to international and national levels by ensuring that children enjoy freedoms and benefits, and before the prevailing need for work, consider rules to classify the work as permissible for infants. On the other hand, education is a right that is often truncated, like child labor, by various factors, in this situation the study deserves importance, since it seeks to explore a relationship between child labor and school drop-out, the work is a start to develop the theme, suggests a study separately between the rural and urban domain of this topic. Finding a relationship between a priori the child population that works and that deserted of education, in the period from 2007 to 2012 in Honduras.
\end{abstract}

Key Words: child labor, children, school dropout, education. 


\section{INTRODUCCIÓN}

En la mayoría de los países en vías de desarrollo, existe una escasa oferta de servicios y una creciente demanda de los mismos, factores que crean un panorama complejo para las sociedades, los pocos proyectos que surgen para menguar las necesidades más básicas, no cuentan con la cobertura deseada, impactando de forma desproporcional en los habitantes de este tipo de países, los cuales para poder beneficiarse de los servicios, deben de pagar agrandando su cuenta.

Siendo los ingresos un elemento necesario para estos fin, se implementan como políticas públicas las estrategias para la reducción de la pobreza, no obstante, la escasa efectividad de las mismas incide en el acrecimiento de la población en estado de pobreza o incluso pobreza extrema como es el caso de muchos países de Latinoamérica, el cual es dramáticamente un indicador más frecuente entre los hondureños.

Como un mal endémico y perenne, la población de estos países aprende a mediar con la privación de algunas necesidades, muchas de ellas básicas. Ante esta situación, y una diversidad de factores adicionales, alcanzan a las familias, viéndose en asediados por cotas que impiden un pleno desenvolvimiento con normalidad en la sociedad, y particularmente en lo que respecta a los niños, quienes se ven envueltos en la necesidad de trabajar, dejando la escuela y pasar a ser un producto de la deserción escolar.

Dentro de un marco cronológico concerniente a los últimos 5 años, se plantea observar que relación existe entre la deserción escolar y el trabajo infantil, mediando a través de otros indicadores vinculados con factores demográficos sociales y económicos de Honduras.

\section{OBJETIVOS}

$\checkmark$ Definir el tipo de relación existente entre las cifras de deserción escolar y el trabajo infantil en el País.

$\checkmark$ Identificar cuáles son los factores comunes entre las dos variables y verificar su relación entre ambas causas. 


\section{TRABAJO INFANTIL}

Vol. 4

E\&A

IIES

164

\subsection{Preámbulo}

Una diversidad de factores económicos, culturales, sociales e individuales, se alimentan unos a otros generando una red que se suele llamar círculo de la pobreza. Su amplitud y profundidad conforman una excesiva complejidad que es difícil de superar (Osorio, 2010).

El trabajo infantil emerge en estos factores como una colaboración al ingreso familiar, con una creciente necesidad de trabajar en diversas actividades, ya sea dentro y fuera del hogar, niños que trabajan en casa para permitir que los miembros de la familia con mayor edad puedan trabajar fuera, que en su mayoría son las niñas, quienes se encargan de las tareas domésticas o cuidar a los más pequeños, sin la supervisión muchas veces de un adulto, quedando en evidencia un problema en que se incurre al momento de solventar el factor de los insuficientes ingresos familiares. Además bajo este mismo factor, los infantes suelen trabajar en diversas actividades para poder alcanzar y mantener un mínimo ingreso para el sostenimiento del hogar(CasaAlianza, 2011).

Los infantes se tornan así, en una mano de obra necesaria, por la situación económica de muchas familias, ya que su situación se agravaría al no contar con los ingresos de los niños. Sin embargo la aportación al ingreso es por lo general bajo (Instituto Nacional de Estadística INE; Secretaría de trabajo y seguridad social STSS; Sistema de información estadística y seguimiento en materia de trabajo infantil SIMPOC, 2003).

La problemática en torno al trabajo infantil, radica en las formas inapropiadas, excesivas o de abuso que atentan contra la integridad física o psicológica de los infantes. Existen una gran cantidad de organizaciones encargadas de velar por el cumplimento de los derechos de los niños, por la eliminación del abuso a menores, por el control y regulación del trabajo infantil, entre muchas otras actividades de protección a la población más vulnerable, los niños. Ante esta situación la organización mundial del trabajo (OIT), se ha propuesto un objetivo visionario para el 2016 (Constance Thomas, OIT, 2011), el cual consiste en erradicar las peores formas del trabajo infantil a nivel mundial.

Por supuesto, no toda actividad que realiza un niño es punible, hay que tener en cuenta que algunas tareas familiares pueden servir para crear y fortalecer 
habilidades y destrezas en el menor. Entonces surge la interrogante ¿Qué es trabajo infantil? ¿Y cuándo éste es inapropiado?

Ante dicha interrogante, el Fondo de Naciones Unidas para la Infancia (UNICEF) define el trabajo infantil como cualquier trabajo que supere una cantidad mínima de horas, dependiendo de la edad del niño o niña y de la naturaleza del trabajo. Este tipo de trabajo se considera perjudicial para la infancia y por tanto debería eliminarse.

Quedan establecidas las siguientes categorías:

$\checkmark$ Entre 5 y 11 años: al menos una hora semanal de trabajo remunerado o 28 horas semanales de trabajo doméstico.

$\checkmark$ Entre 12 y 14 años: al menos 14 horas semanales de trabajo remunerado o 28 horas semanales de trabajo doméstico.

$\checkmark$ Entre 15 y 17 años: al menos 43 horas de trabajo remunerado o de trabajo doméstico semanales.

UNICEF también ha desarrollado un conjunto de criterios básicos para determinar cuándo el trabajo infantil es inapropiado: (CIAF, Ana Osorio, Gloria Ricardo, Diana Ramirez, 2010):

E\&A

IIES

165

$\checkmark$ Es con dedicación exclusiva a una edad demasiado temprana

$\checkmark$ El trabajo provoca estrés físico, social o psicológico indebido

$\checkmark$ Se trabaja y se vive en la calle en malas condiciones

$\checkmark$ El salario es inadecuado

$\checkmark$ El niño tiene que asumir demasiada responsabilidad

$\checkmark$ El trabajo impide el acceso a la escolarización

$\checkmark$ El trabajo mina la dignidad y autoestima del niño

$\checkmark$ Impide conseguir un pleno desarrollo social y psicológico

Las condiciones de pobreza, la falta de protección social, la desintegración familiar, programas de reubicación y escasez de vivienda, poca motivación y acceso a la educación, conforman la situación actual de la sociedad hondureña que inducen a una gran cantidad de niños y niñas a incorporarse al mercado de trabajo para así colaborar económicamente en sus hogares y lograr satisfacer las necesidades básicas de su familia. 
Vol. 4

E\&A

IIES

166

\subsection{Antecedentes del Trabajo Infantil en Honduras}

Un objetivo fundamental de todas las naciones y organismos internacionales es la protección, cuidado y educación de la niñez. Honduras no es la excepción, a través de la historia se ha desarrollado un marco legal que contempla los derechos de los niños y vela por su protección, siendo prioridad la salud, bienestar y educación de los mismos. Algunos elementos de este marco legal se detallan a continuación:

1. En Honduras, debido al poco desarrollo industrial, ha sido un país que se ha dedicado a la producción agrícola, por lo que ha sido normal que una gran cantidad de niños y niñas aprendan a temprana edad las tareas del campo, para colaborar con sus padres a contribuir al sustento de la familia. Anteriormente, no existía la diversidad en el mercado laboral, ni la variedad de educación académica o técnica de hoy, por lo tanto la mayoría de las actividades laborales se limitaban a la zona rural. Antes de la Huelga de 1954, era común encontrar menores de edad realizando tareas en meloneras y bananeras. La inexistencia de un Código de Trabajo en Honduras antes de 1959 permitió la explotación indiscriminada de menores en casi todas las actividades.

2. Otro logro se ha dado en el año 1982 cuando en la constitución de la república se establece que el Estado tiene la obligación de brindar especial protección a los niños y niñas cuyos padres estén imposibilitados económicamente para satisfacer sus necesidades básicas y su educación. Así mismo se establece en el artículo 124 lo siguiente:

> "Todo niño debe ser protegido contra toda forma de abandono, crueldady explotación. No será objeto de ningún tipo de trato".

> "No deberá trabajar antes de una edad mínima adecuada, ni se le permitirá que se dedique a ocupación o empleo alguno que pueda perjudicar su salud, educación, o impedir su desarrollo físico, mental o moral".

3. Se prohíbe la utilización de los menores por sus padres y otras personas, para actos de mendicidad. La Secretaría del Trabajo y Seguridad Social (STSS) implementó en el 2001 el Reglamento sobre Trabajo Infantil en Honduras, donde se describen algunas actividades laborales que se consideran nocivas para la salud física y mental de los niños de Honduras.

\footnotetext{
9 Mediante el Acuerdo Ejecutivo N ${ }^{\circ}$ STSS-211-01, la STSS incorporó a la ley nacional una serie de términos en los que se establece los criterios para considerar el trabajo del infante como nocivo.
} 


\subsection{Diagnóstico del Trabajo Infantil en Honduras}

Con base a los datos que proporciona la Encuesta Permanente de Hogares (EPHPM), que realiza el Instituto Nacional de Estadística (INE), en lo concerniente al período de los últimos 5 años, la población total se ha ido incrementando desde 7,529,403 personas en el año 2007, hasta 8,303,399 hondureños. Sin embargo, se observa que la población infantil que comprende el rango de edad entre 5 y 17 años, curiosamente, ha ido menguando, desde un $38 \%$, a un 31\% de la población total nacional para el año actual (2012).

La diferencia entre el sector rural y urbano respecto a la población total corresponde un $9 \%$, en cambio entre los infantes la diferencia es de 20 puntos porcentuales, indicando que el área rural tiene más concentración de la población y que los nacimientos son a su vez más comunes en las zonas menos urbanizadas.

El trabajo infantil en honduras entre los años 2007 y 2012 varia de $14.5 \%$ a $13.5 \%$, con un descenso total de $1 \%$ a lo largo de este periodo, pero sin un comportamiento monótono, ya que en el año 2008 el porcentaje de menores que trabajan es de $13.1 \%$ y un año más tarde, en el 2009 , ocurre un incremento hasta alcanzar el valor máximo $14.7 \%$, como consecuencia quizá a la medida interpuesta por el gobierno central, en la cual el presidente Manuel Zelaya incrementó el salario mínimo, generando despidos y cierre de algunas pequeñas empresas, promoviendo a muchas familias a distribuir la carga para el sostenimiento del hogar, regulándose la situación con el pasar del tiempo y a partir de aquí se observa una tendencia hacia la baja (ver Figura 3.1), implicando que la cantidad de niños que trabajan ha ido disminuyendo desde entonces.

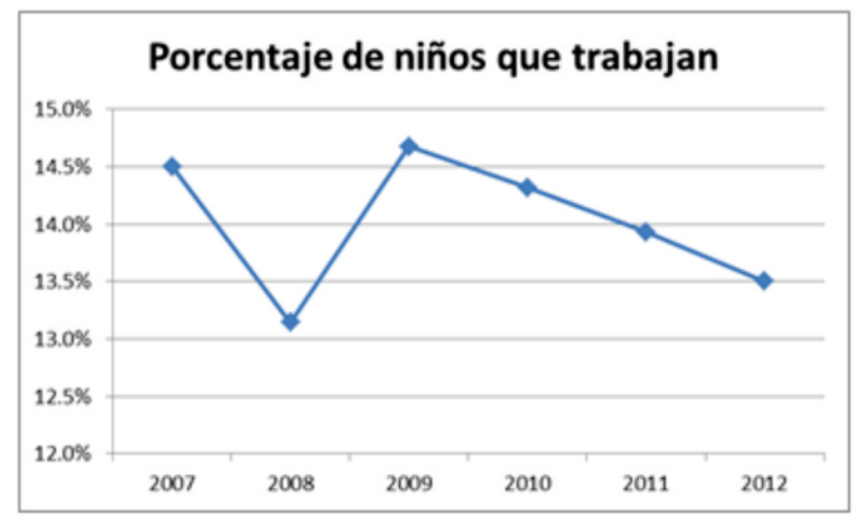

Figura 3.1 Porcentaje de niños que trabajan en Honduras

Fuente: EPHPM 2007-2012 
Vol. 4

E\&A

IIES

El promedio de las tasas de trabajo infantil en los años de estudio, es de $14.01 \%$, con un coeficiente de variación de 0.042 , que establece que las cifras se comportan muy similares a lo largo de este periodo. De la misma forma, existe homogeneidad en los indicadores de trabajo infantil por dominio urbano y rural, donde promedian $8.7 \%$ y $17.6 \%$ respectivamente, los cuales representan la relación entre los niños que trabajan en el dominio con respecto al número de infantes en el mismo. Evidenciando que es más común que los niños se incorporen en las actividades laborales si son de la zona rural, ya que por cada dos niños que trabajan del área rural, tan solo un niño del área urbana se encuentra en actividades laborales.

Actualmente se encuentran aproximadamente 351,522 niños que trabajan dentro del país, de los cuales el 76\% pertenecen al dominio rural, porcentaje que no ha manifestado variaciones significativas en los últimos 5 años (Ver Figura 3.2), implicando que las actividades agrícolas continúan siendo las más comunes de Honduras y donde la mayoría de las tareas incorporan a los niños por su menor preparación académica.

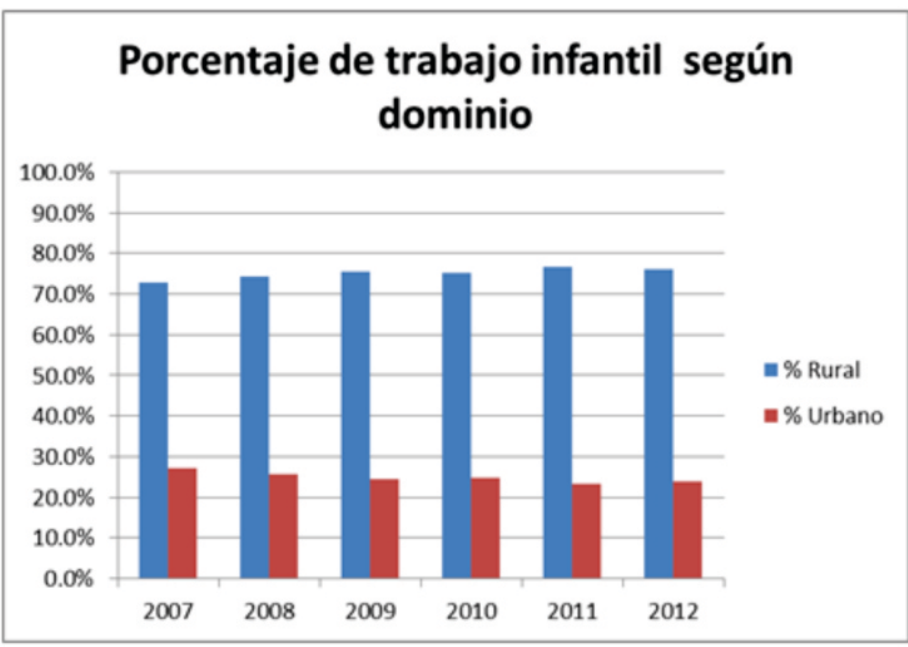

Figura 3.2. Porcentaje de trabajo infantil según dominio Fuente: EPHPM 2007-2012 
Adicionalmente a la carga laboral que emprenden los menores del país, algunos de ellos llevan el estudio como otra actividad, bajo esta característica se encuentran 121,928 niños que representan el $34.7 \%$ del total de menores que laboran. Siendo víctimas de la exigencia de trabajo, estos pequeños pueden incidir en la deserción escolar. Lamentablemente el $65.3 \%$ restante lo conforman niños que se dedican exclusivamente a trabajar, habiendo dejado a un lado la escuela y pasando a ser efecto de la deserción escolar o posiblemente nunca fueron parte del sistema educativo.

\section{DESERCIÓN ESCOLAR EN HONDURAS}

\subsection{Reseña de la Educación en Honduras}

La educación en Honduras ha sufrido grandes cambios en el devenir del tiempo. En la época colonial, era la iglesia católica la que estaba a cargo de la educación del pueblo indígena, que tenía como primer objetivo brindarles una educación básica, enseñándoles el idioma castellano para posteriormente dedicarse de una forma exhaustiva a la "evangelización de los indios".

Durante mucho tiempo solo las clases privilegiadas tenían acceso a los sistemas educativos, hoy en día la constitución de la república establece en el artículo 171 que la educación impartida oficialmente será gratuita y la básica será además, obligatoria y totalmente costeada por el Estado.

Sin embargo, la pobreza es un flagelo que azota fuertemente a Honduras y uno de los sectores más afectados es la niñez. La precaria situación económica y política que enfrenta el país incide negativamente en el sistema educativo, por lo que miles de niños y niñas no pueden gozar plenamente de su derecho a una educación integral.

El estado es el encargado de crear y regular políticas públicas eficaces orientadas a aumentar la cobertura educativa, reducir los niveles de analfabetismo, organizar, dirigir y supervisar todos los procesos concernientes a la educación formal y no formal del país. 
Vol. 4

E\&A

IIES

170

\subsection{Actualidad de la Deserción Escolar en Honduras}

La deserción escolar ha sido un problema de mucha preocupación en Honduras, ya que el sistema de educación presenta muchas dificultades, como la insuficiente cobertura educativa, pocos estímulos a la permanencia de la población estudiantil, así también la repitencia y el retraso escolar, fenómenos que con frecuencia anteceden a la deserción escolar.

Este problema unido a un bajo nivel de aprendizaje de los contenidos básicos de la enseñanza, se convierte en un atentado contra el aprovechamiento del potencial de los niños y niñas desde temprana edad y sus efectos negativos se acumulan a través del tiempo lo que incide de manera desigual en las oportunidades de bienestar de los sectores más pobres.

Las causas para que los estudiantes se ausenten de los centros educativos pueden ser diversas, en el caso de los sectores menos favorecidos, los jóvenes muy frecuentemente se ven obligados a abandonar o pausar sus estudios para incorporarse en actividades laborales y generar un ingreso extra para el sostenimiento de su familia. Sin embargo, otros factores como la falta de motivación, el alcoholismo, la desintegración familiar, difícil acceso a los centros educativos, entre otros, pueden incidir negativamente en el rendimiento académico de los niños induciendo finalmente al menor a abandonar su educación. La deserción escolar es un problema cuya responsabilidad no es totalmente del estudiante, por lo que todos debemos contribuir a disminuir la deserción escolar, la cual demanda muchos esfuerzos de las autoridades, del sistema educativo y de la sociedad en general.

Los efectos negativos de la deserción escolar son tan graves que la CEPAL (comisión económica para América latina), en su edición del panorama social de América latina ha señalado lo siguiente, "este es quizás el principal escollo que los sistemas educativos de la región debieran salvar para desempeñar con más plenitud y eficacia su papel igualador de oportunidades y de inclusión social”. Hemos entrado al siglo 21 y a pesar de los esfuerzos realizados, el problema de la deserción escolar continua, lo que debe constituir una gran preocupación de todos los sectores del país, que nos conduzca a unir todas nuestras fuerzas para enfrentar con firmeza este drama que amenaza el sistema educativo nacional, contrarrestando la primera tendencia de abandonar la escuela, para engrosar el circulo de pobreza y marginalidad y trabajo infantil. 


\subsection{Diagnóstico de la Deserción Escolar en Honduras}

El presente diagnóstico de la deserción escolar se basa en los datos obtenidos a partir de la encuesta permanente de hogares realizada por el INE correspondiente a los años del 2007 al 2012.

La taza de matrícula de los estudiantes en los distintos centros educativos del país en todos los niveles se mantiene de forma estable durante el periodo anteriormente establecido, factor que resulta de grata satisfacción ya que a pesar de los distintos acontecimientos que han podido incidir en el no ingreso de estudiantes a los centros de estudio, con lo cual podemos aseverar que el deseo de seguir formándose académicamente es un elemento que destaca en la población infantil hondureña.

MATRICULA POR AÑO DE ESTUDIO

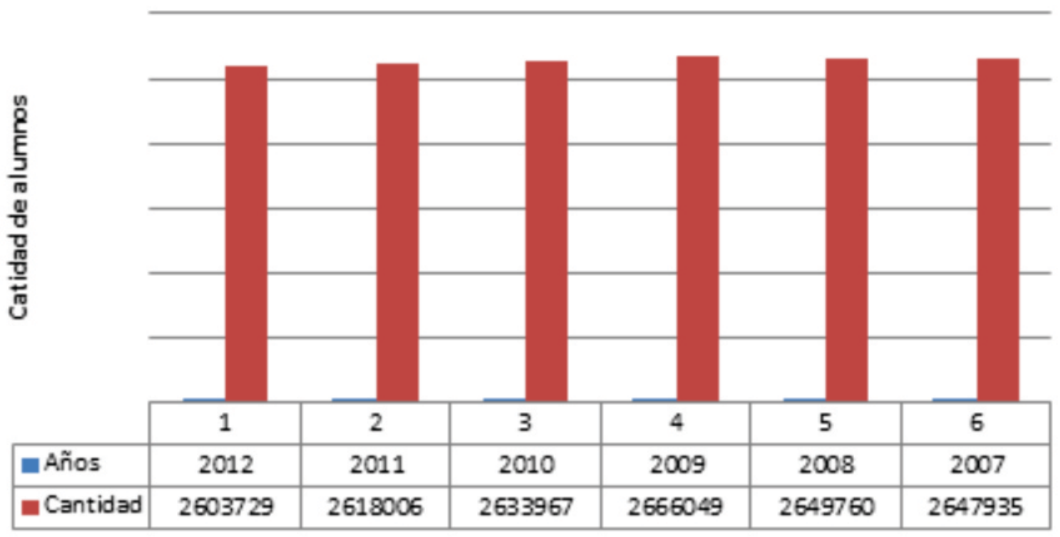

E\&A

IIES

Figura 4.1. Matrícula escolar

Fuente: EPHPM 2007-2012

Del total de estudiantes matriculados en el sistema educativo nacional aproximadamente el $54 \%$ pertenecen al área rural, dato que se ha mantenido a lo largo de los años sobre el que abarca el estudio realizado, esto se debe entre otros aspectos a que la mayoría de la población hondureña reside en esta zona, de este total más del $50 \%$ corresponde a la población masculina lo que nos hace plantearnos las siguientes interrogantes: ¿Qué factores influyen para que el sector 
Vol. 4

E\&A

IIES

172

masculino sea el de mayor afluencia en el sector académico? ¿ ¿A qué edad la población acude más a los centro educativos?

Realizando un análisis retrospectivo comenzaremos observando que para el año 2007 el porcentaje de ingresos al sector educativo correspondiente al área urbana es de $57 \%$ de este porcentaje un poco más de la mitad son hombres de los que en su mayoría oscilan entre los 6 y 11 años de edad, con lo que de acuerdo al rango de edades estos corresponde al sector de educación primaria.

Para el 2008 las cifras reflejan que la mayoría de los estudiantes que ingresaron pertenecen a la zona urbana de los cuales solo el $66 \%$ de las personas que se inscriben asiste a clase hecho que debe llamarnos a reflexionar ¿Qué sucede con la educación en la niñez hondureña? ¿Que provoca que más del $40 \%$ de ellos abandonen los salones de clases? ¿Que motiva esta lamentable situación? en el año 2009 aunque poco se logró un aumento en el porcentaje de las personas que asisten a clases llegando este a un $68 \%$ dato que se ha mantenido de manera casi constante a lo largo de todos estos años hasta llegar al año 2012 donde se refleja un aumento en la asistencia a clases con un porcentaje del $70 \%$; indagando un poco en los elementos que giran en torno a este fenómeno tenemos que hoy en día hay más universalidad y promoción por parte de medios que motivan el deseo de educación, la necesidad de prepararse ante la falta de oportunidades genera mayor interés al ingresar al sistema educativo por parte de la población y la cobertura que cada vez se vuelve más amplia llegando a sectores que años atrás se pudo pensar que jamás tendría acceso.

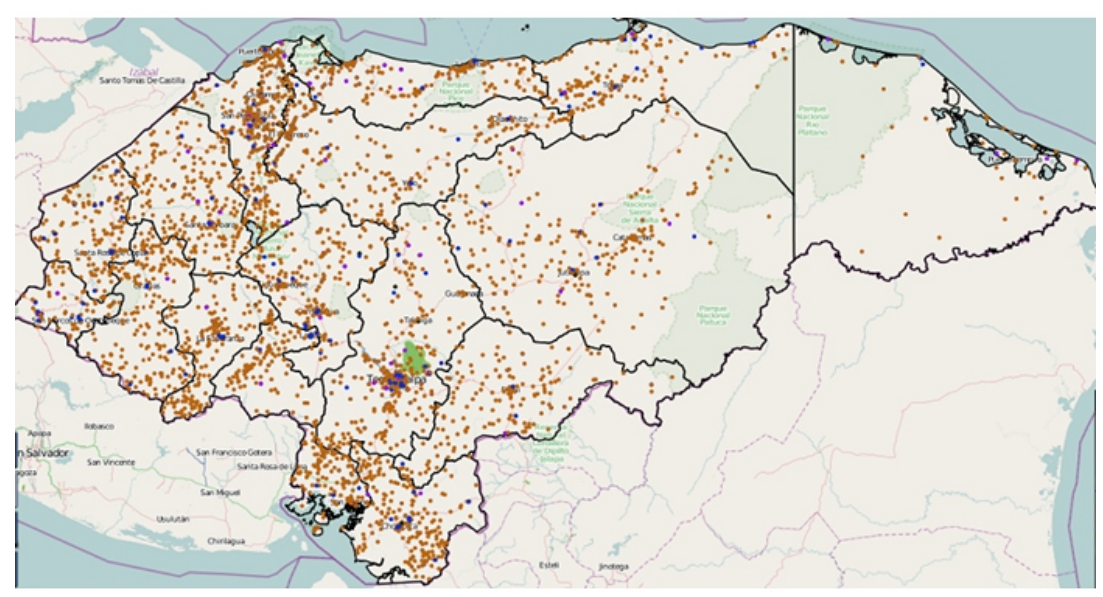

Figura 4.2. Cobertura de centros de estudio escolar

Fuente: Secretaria de educación 
Por otro lado si nos enfocamos en el área rural notaremos que el porcentaje de personas que ingresan a los centro educativos y no desertan ha ido aumentando gradualmente hasta alcanzar una anotación máxima del 55\% aunque es más de la mitad es importante recordar que en el área rural hay más población por ende el porcentaje de desertores escila entre el $10 \%$ hecho que resulta verdaderamente lamentable en nuestro país.

Entre los factores más comunes o de mayor énfasis son: la ausencia de servicios de educación primaria adecuadas la situación económica relacionada con el trabajo infantil, costos de la escolarización para los padres de familia, problemas de salud y alimentación así como mecanismos de exclusión, entre otros.

Todo lo anteriormente mencionado deja como consecuencia que un alto índice de estudiantes decida desertar.

De acuerdo con las estadísticas relacionadas con la deserción escolar nos damos cuenta ha cada año va disminuyendo este porcentaje, aunque sigue siendo un problema permanente en la educación de nuestro país.

Se estima que el año 2007 hubo un $0.8 \%$ de niños desertaron de los centro educativos, y comparamos el año 2008 donde el país tuvo conflictos políticos los cuales obligaron a muchos estudiantes a retirarse de las escuelas donde la estadística ascienda alcanzando un $1.41 \%$ de deserción, para el año 2009 la reducción de la deserción se ve reflejada alcanzando un aproximado del 1.18\% sobre el caso, el años 2010 nos trae mejores noticias y este fenómeno sigue bajando hasta alcanzar $0.85 \%$ de retiros de niños de las escuelas, en el 2011 aun los porcentajes son más alentadores porque el porcentaje estimado llega al $0.49 \%$, no así el presente año 2012 donde se refleja que este problema ascendió al reflejar una estimado del $0,64 \%$ de niños que abandonan las escuelas.

\section{RELACIÓN ENTRE TRABAJO INFANTIL Y DESERCIÓN ESCOLAR}

En honduras los índices de pobreza son alarmantes, la sociedad civil, el gobierno central y otros organismos internacionales han emprendido un trabajo conjunto, con la finalidad de reducir los índices de la pobreza, como objetivo en el nuevo milenio, entre enero del 2000 y mayo de 2001, se formalizó la estrategia de reducción de la pobreza interpuesta en el país. En la primera década del milenio

E\&A IIES 
Vol. 4

E\&A

IIES

174

2000-2009, se obtuvo resultados positivos, reduciendo el porcentaje de pobres desde un $70 \%$ de la población total hasta un se presenta

\subsection{Factores que Influyen en el Trabajo Infantil}

Como mencionamos anteriormente el trabajo infantil es un factor que surge de una multiplicidad de factores, bajo esta premisa, se analiza algunas de las dimensiones como son el contexto demográfico, económico y social.

Para nuestro efecto, consideramos complementariamente el comportamiento del trabajo infantil sobre estos dos dominios. Para poder diferenciar adecuadamente las actividades en las cuales se ven involucrados los niños y niñas de Honduras. Con el fin de categorizar el trabajo generado en los sectores productivos del país.

El Contexto económico, permite separar explícitamente las actividades desempeñadas por los trabajadores, en este particular caso, sobre la población de menores de 18 años.

Desde el punto de vista económico, se inicia el estudio en el factor financiero preponderante para el trabajo, la pobreza, dado que no es posible hacer un estudio objetivo con una variable ingreso de mucha variabilidad, consideramos tomar una variable superlativa que la comprenda, la pobreza se define a partir del conjunto de necesidades insatisfechas, y se establece en la EPHMP solamente sobre los hogares que declaran ingresos, siendo un indicador tangible y centralizado del ingreso por hogar.

La canasta básica, es el elemento más característico de las necesidades de una población, ya que prioriza en la escala de lo ineludible

\section{INDICADORES}

\section{Nivel de pobreza}

Es una situación o forma de vida que surge como producto de la imposibilidad de acceso o carencia de los recursos para satisfacer las necesidades físicas y psíquicas básicas humanas que inciden en un desgaste del nivel y calidad de vida de las personas, tales como la alimentación, la vivienda, la educación, la asistencia sanitaria o el acceso al agua potable. También se suelen considerar la falta de medios para poder acceder a tales recursos, como el desempleo, la falta de ingresos o un nivel bajo de los mismos. También puede ser el resultado de procesos de exclusión social, segregación social o marginación. 


\section{Canasta básica (costo)}

La canasta básica alimentaria (CBA) se conoce como el conjunto de alimentos, expresados en cantidades suficientes para satisfacer las necesidades de calorías de un hogar promedio. Sin embargo, debe quedar claro que representa un mínimo alimentario a partir de un padrón de consumo de un grupo de hogares de referencia y no una dieta suficiente en todos los nutrientes. Por lo tanto la canasta básica alimentaria no es una dieta ideal y, en consecuencia no debe ser utilizada como instrumento para la educación alimentaria nutricional, ni para establecer necesidades alimentarias de un individuo o una población en particular

\section{DESERCIÓN}

La deserción escolar es el abandono temporal o definitivo que efectúa una persona, de sus estudios escolares formales. Como consecuencia, se genera un retraso en el proceso de alfabetización. Entre las causas más comunes, se encuentran los escasos recursos económicos y la desintegración familiar (Ramirez, 2010).

\section{ANÁLISIS}

Consideramos los indicadores expresados anteriormente:

$\checkmark$ Pobreza

$\checkmark$ Canasta básica

$\checkmark$ Desertores

$\checkmark$ Trabajo infantil

\section{Primer modelo planteado}

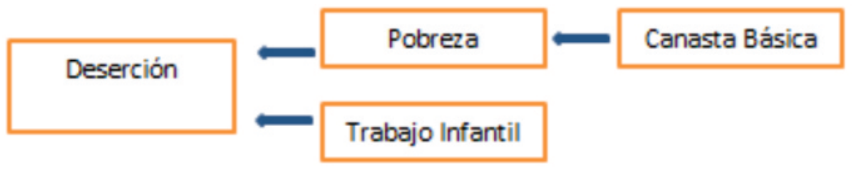

Figura 5.1. Primer Modelo de relaciones

Dado que la canasta básica, pese a presentar dos valores distintos según el dominio, siendo mayor en el área urbana que en la rural, comprende las necesidades de un hogar y la pobreza se interpreta de manera práctica, como la incapacidad de satisfacer las necesidades básicas así que de forma contextual, eliminamos la variable CANASTABÁSICA. 
Vol. 4

E\&A

IIES

Complementariamente a este razonamiento realizamos la prueba de hipótesis sobre el modelo de regresión lineal, donde el valor-p sugiere que dicha variable no supone relación para la deserción

\section{Segundo modelo planteado}

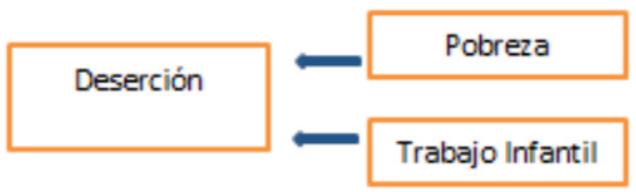

Figura 5.2. Segundo Modelo de relaciones

Trabajo InfantilSin embargo el coeficiente de determinación no es el apropiado, por lo que se procede a encontrar otro nuevo modelo y el cual podemos establecer a partir de la siguiente ecuación:

Deserción $=0.032149 *$ Trabajo Infantil

Modelo 5.1

Donde

$\boldsymbol{R}^{\wedge} 2=0.88521$,

Valor Crítico $F=8.66 \mathrm{E}+08$,

Valor- $p=0.00158247$

Modelo que permite establecer la siguiente relación, por cada 31 niños que trabajan, un niño deserta de la escuela.

Por otra parte el dominio tiene una influencia sobre la frecuencia de que un menor se incorpore en tareas como parte del trabajo infantil. Por lo que establecemos un modelo según el dominio, consideramos trabajo infantil y deserción por área rural y urbana. (ver tablas 5.1 y 5.3 ) 
Tabla 5.1 Dominio urbano, total de infantes que trabajan y desertores en el rango de años 2007-2012

\begin{tabular}{|r|r|}
\hline \multicolumn{2}{|c|}{ Urbano } \\
\hline Trabajan & Desertores \\
\hline 111,810 & 2,803 \\
\hline 89,580 & 6,457 \\
\hline 95,578 & 5,133 \\
\hline 93,232 & 4,918 \\
\hline 83,418 & 1,886 \\
\hline 83,418 & 2,603 \\
\hline
\end{tabular}

Fuente: EPHPM 2007-2012

Estableciendo así el siguiente modelo para el dominio Urbano:

Deserción $=\mathbf{0 . 0 4 2 2 9 9 2} *$ Trabajo Infantil $\quad$ Modelo 5.2

Donde

$\boldsymbol{R}^{\wedge} \mathbf{2}=0.649966664$,

Valor Crítico $F=0.00602195$,

Valor- $p=0.00315057$

Modelo que nos permite concluir que por cada 24 niños que trabajan en la zona urbana, 1 desierta de la escuela.

Mientras para el dominio Rural, tenemos la serie temporal concerniente al mismo periodo de tiempo anterior (2007 al 2012).

A lo largo del estudio se maneja que una consecuencia de la pobreza es el trabajo infantil, como prueba de la aseveración planteamos la comparación de los hogares pobres y el trabajo infantil. La serie histórica en el lapso de tiempo (2007-2012) presenta los datos en la tabla 5.5: 
Vol. 4

E\&A

IIES

Tabla 5.3 Hogares pobres y total infantes trabajadores rango 2007-2012

\begin{tabular}{|r|r|}
\hline \multicolumn{1}{|c|}{$\begin{array}{c}\text { Hogares } \\
\text { Pobres }\end{array}$} & $\begin{array}{l}\text { Trabajo } \\
\text { Infantil }\end{array}$ \\
\hline 936,722 & 410,290 \\
\hline 976,710 & 348,250 \\
\hline 991,763 & 391,195 \\
\hline $1,038,864$ & 377,158 \\
\hline $1,064,166$ & 359,617 \\
\hline $1,206,698$ & 351,522 \\
\hline
\end{tabular}

Fuente: EPHPM 2007-2012

Al buscar un modelo lineal que medie la relación existente entre ambas categorías se obtuvo:

El modelo lineal resultante establece

Trabajo Infantil $=0.35650115 *$ Hogares Pobres

Modelo 5.4

\section{Donde}

$\boldsymbol{R}^{\wedge} \mathbf{2}=0.78344423$,

Valor Crítico $F=6.6516 \mathrm{e}^{\wedge}-5$,

Valor $-p=1.2046 \mathrm{e}^{\wedge}-5$

Lo que concluye con la relación que por cada 3 hogares pobres, existe un niño que trabaja.

\section{CONCLUSIONES}

$\checkmark \quad$ A nivel mundial, el trabajo infantil es un fenómeno amplio, complejo y multicausal. La carencia de información confiable y de análisis cuantitativos y cualitativos dificulta que se encuentren formas efectivas de afrontar el problema. Por muchos años, la falta de información sobre sus causas, magnitud, naturaleza, y consecuencias, ha sido un considerable obstáculo para llevar a cabo una acción eficaz de cara a enfrentar, detener y eliminar este fenómeno que afecta a millones de niños. 
La mayor parte de la niñez hondureña acude al sistema educativo. La mayor cobertura se logra en la educación primaria y va reduciéndose conforme aumenta la edad. Sin embargo, la mayor parte de la niñez trabajadora en actividades económicas hondureña no asiste a un centro educativo formal. La población infantil ocupada que asiste a la escuela en un gran porcentaje también realiza quehaceres del hogar. Es difícil bajo esta realidad determinar la disponibilidad de tiempo de estos niños y niñas para disfrutar su niñez fuera de la escuela, el trabajo y los quehaceres domésticos. La información de la encuesta de hogares no profundiza en este aspecto, pero es notorio que queda en efecto muy poco tiempo disponible para actividades recreativas, de esparcimiento, y el descanso.

\section{RECOMENDACIONES}

$\checkmark$ Para la disminución del trabajo infantil es necesario que el Gobierno emprenda políticas y acciones en pro del apoyo de las familias, principalmente de aquellas que se encuentran vulnerables.

$\checkmark$ Concientizar a los padres de familia, sobre la importancia de la educación de sus hijos, que a futuro tendrán una mayor diversidad de oportunidades laborales.

$\checkmark$ Utilizar nuevas técnicas de enseñanza para reducir el grado de complejidad de aprendizaje en el alumno y así reducir la repitencia, evitando que el padre de familia motive al alumno al abandono de las clases.

$\checkmark$ Investigar los casos reportados de lesiones y enfermedades serias ocasionadas por el trabajo infantil, y promover mejores condiciones de seguridad que reduzcan su incidencia, así como estudiar las consecuencias a largo plazo del trabajo sobre el bienestar físico de los niños y niñas trabajadoras.

$\checkmark \quad$ El gobierno debe tener un plan para reducir las ausencias de los centros escolares, en el caso de los sectores menos favorecidos, donde los jóvenes tienen que ayudar a sus progenitores a buscar el sustento diario. 
Vol. 4

E\&A

IIES

\section{BIBLIOGRAFÍA}

Casa Alianza. (2011). El trabajo infantil en Honduras, Diagnostico situacional de algunas de las peores formas de trabajo infantil en el país.

CIAF, Ana Osorio, Gloria Ricardo, Diana Ramirez. (2010). Trabajo infantil: Un círculo vicioso que perpetúa la pobreza.

Constance Thomas, OIT. (2011). El estado actual del trabajo infantil.

Gobierno de Honduras. (2001). Estrategia para la reducción de la pobreza, un compromiso de todos por Honduras.

Instituto Nacional de Estadística INE; Secretaría de trabajo y seguridad social STSS; Sistema de información estadística y seguimiento en materia de trabajo infantil SIMPOC. (2003). Análisis cualitativo de la situación del trabajo infantil en Honduras.

Instituto Nacional de Estadística INE. Encuesta Permanente de Hogares.

OIT. (s.f.). Organización Internacional del Trabajo. Recuperado el 11 de septiembre de 2011, de http://ilo.org/ipec/areas/Childdomesticlabour/lang-es/index.htm

Osorio, C. (2010). Círculo vicioso de la pobreza. Revista digital Innovación y experiecnias educativas, 1-8.

Ramirez, Y. (2010). La calidad de la gestión educativa y la deserción escolar del nivel primario en la Institución Educativa Brigada Silvia de Ochoa Chorillos . Lima, Perú.

Secretaria de Educación, http://www.se.gob.hn/ capturado septiembre 2011 